\title{
EL CONTEXTO Y EL CENTRO RESIDENCIAL PARA LAS PERSONAS ADULTOS MAYORES EN COLOMBIA Y ESPAÑA. LA EMPRESA SOCIAL UNA ALTERNATIVA PARA EL BIENESTAR
}

\author{
POR \\ Lourdes OSORIO BAYTER ${ }^{1}$ y \\ Francisco SALINAS RAMOS ${ }^{2}$
}

\section{RESUMEN}

La protección a los adultos o personas mayores se ha convertido en una política de Estado, esto debido al creciente registro de vulneración de los derechos de los mismos. Por lo tanto, conocer estas políticas gubernamentales y sociales que se encuentran vigentes, también el actual sistema de seguridad social y su cobertura para ésta población tanto en España y Colombia, así como su contexto legal y social, además de identificar los espacios donde un adulto mayor encuentra la alternativa de vivir y compartir sus años longevos, ya sea en los llamados hogares geriátricos, residencias o centros de atención a mayores, como también el papel que las empresas solidarias juegan en la oferta y demanda de un mercado donde el segmento del adulto mayor de acuerdo a las necesidades en este contexto, genera mayores demandas de productos y profesionales especializados con sentido social. Este tejido de relaciones en pro del Bienestar hacía el adulto mayor, conlleva a presentar los resultados parciales de una investigación realizada en hogares o residencias para personas mayores ubicados en Ávila, Madrid y Santiago de Cali.

Palabras Clave: envejecimiento, personas mayores, residencias, hogar geriátrico, bienestar social, política social, empresas solidarias.

Claves ECONLIT: A100, E270, J110, J140.

\footnotetext{
${ }^{1}$ Universidad Autónoma de Occidente. Dirección de correo electrónico: leosorio@uao.edu.co.

${ }^{2}$ Universidad Católica de Ávila. Dirección de correo electrónico: francisco.salinas@ucavila.es.

REVESCO No 121 - Segundo Cuatrimestre 2016 - ISSN: 1885-8031 - www.ucm.es/info/revesco

http://dx.doi.org/10.5209/rev_REVE.2016.v121.51307

Fecha de recepción: 03/02/2015

Fecha de aceptación: 05/10/2015
} 


\title{
THE CONTEXT AND THE RESIDENTIAL CENTER FOR THE ELDERLY IN COLOMBIA AND SPAIN. THE SOCIAL ENTERPRISE ONE ALTERNATIVE FOR WELFARE
}

\begin{abstract}
The protection to the older people or elderly adults (EA) it has turned in a state policy, there is an increased number of registers due to the violation of these rights. Due to this it is important to be informed about social politics that are still being used and to the types of populations it is applied to, referring to Spain and Colombia in social and legal aspects. Also, identity the spaces where an elderly adults finds an alternative to live and share their elderly age is the geriatric homes, residencies or centers of attention to the older, as also the role that the solidarities companies are playing in the offer and demand in a market where the target of the elderly adults, agree with their necessities in this contexts generates a bigger demand of products and specialized professionals with social sense. This tissue of relations in favor the well-being toward elderly adults s carries to presenting the partial results of an investigation realized in homes or residencies to elderly adults situated in Ávila, Madrid and Santiago de Cali.
\end{abstract}

Keywords: aging, elderly, nursing, nursing home, social welfare, social policy, Social enterprise.

\section{INTRODUCCIÓN}

Según estudios demográficos de Naciones Unidas (2002), los adultos mayores $\left(\mathrm{AM}^{3}\right)$ son el grupo que crece más rápidamente en la población. En el año 2000 el mundo tenía 590 millones de personas de más de sesenta años de edad y para el año 2025 se proyectan 1100 millones, con un aumento del $224 \%$ en relación con las cifras de 1975, es decir que el crecimiento de esta población en el año 2025 llegará a ser cuatro veces mayor a lo esperado. Esta traslación social, no puede pasar desapercibida por los poderes públicos ni por las empresas sociales, puesto que aquí se vislumbra un gran potencial de mercado para el presente y el futuro inmediato.

\footnotetext{
${ }^{3}$ En el artículo se utilizará indistintamente las expresiones "adulto mayor" y "persona mayor", el primero más usado en Colombia y el segundo en España, identificado con la sigla: AM.
} 
La información que se presenta a continuación forma parte de los resultados de estudios realizados por los autores, esencialmente en una perspectiva política, legal y social comparativa en Ávila, Madrid (España) y Santiago de Cali (Colombia), enfocado al contexto de los AM y las residencias para adultos mayores, en adelante RAM, de estratos socioeconómicos 3, 4 y 5 (medio y alto).

Algunos de los datos que describiremos, forman parte de los resultados de uno de los estudios $^{4}$ efectuado entre el 2013 y 2014, con una muestra de 27 residencias en Santiago de Cali (la mayoría fundaciones), 8 en Madrid y 4 en Ávila (las 12 pertenecientes a la Federación Lares), donde se aplicaron encuestas a residentes (60 en Cali) y a 30 familiares del AM (18 en Cali y 12 en Madrid y Ávila), además de 34 entrevistas en profundidad a encargados o administradores de RAM (22 en Cali y 12 en Madrid y Ávila). Igualmente, a través de la recolección de información secundaria, en el presente documento abordaremos un esbozo tanto del marco institucional de las políticas sociales y la protección social hacía la población del AM en ambos países, como también, acerca del bienestar de éste segmento, según la estructura organizacional predominante en los hogares que lo acogen. Por último, se cierra con un apartado que muestra la importancia de la empresa de estructura social (cooperativas, fundaciones, otras) y su incidencia en la calidad de vida de éste grupo poblacional.

El interés por el tema del AM, su relación con las políticas sociales y la empresa social, se debe a que éste segmento creciente y con poder adquisitivo, pueden ser hoy en día para las empresas, las nuevas estrellas del mercadeo para el entorno empresarial, ya sea por los productos que se adquieren en las residencias especializadas para ellos o porque se demanda mayor número de servicios según la estructura organizacional, siendo así un claro mercado objetivo identificable para las empresas sociales considerando el acelerado crecimiento de esta población. Claro está, que también depende del contexto político-social donde estén ubicados.

\footnotetext{
4 "Variables de Influencia en la Preferencia y Lealtad de Marca, en Residencias de Adultos Mayores. Un Análisis Social Comparativo Entre Ávila-Madrid (España) y Santiago de Cali (Colombia)". 2013-2014. Documento que pretende entre otros: a) Analizar la realidad social y económica de los AM de Cali, Madrid y Ávila; b) Informar sobre los servicios sociales y asistenciales ofrecidos al AM en las residencias de ambos entornos y c) Conocer los comportamientos del AM ante determinadas imposiciones de marcas. Estudio exploratorio que aborda 4 públicos con entrevistas en profundidad y encuestas: a) Profesionales especializados de las Residencias; b) Familiares del AM de las Residencias, c) Personas AM residentes en los Centros y d) Expertos en publicidad. Aplicado en Residencias de estrato socioeconómico 3, 4, 5 y 6 de tres ciudades de Colombia y España.
} 
Es así como, generar estrategias de mercadeo innovadoras y llamativas para éste segmento se convierte en una propuesta competitiva en el entorno del mercado; lo que justifica investigar más profundamente sobre el macro y micro entorno de este nicho. Adentrándonos en el contexto de la economía social, en los servicios de bienestar social y en particular el sector socio-sanitario, que para efectos de la investigación realizada y que abordamos en éste artículo mostrando parte de los resultados, conllevará muy seguramente, a la toma de decisiones o aprendizaje de la adecuada gestión de las viviendas/residencias para los AM, así mismo las federaciones del tercer sector o las asociaciones de cooperativas ven como una oportunidad de fortalecimiento y promoción hacia dicho segmento de población. También, los profesionales encargados de señaladas estructuras, podrán hacer útil la información resultante de éste documento, ya sea para intervenir de manera integral en el AM en relación a los servicios que prestan en pro de las deseos, necesidades y preferencias del AM y sus familiares; así como en la forma de organización (colaborativa o de asociación) según las características del entorno, para un aprovechamiento más óptimo de éste segmento, su experticia y la proyección de una mayor calidad de vida de los mismos.

\section{LA PERSONA ADULTO MAYOR Y SU CONTEXTO}

El crecimiento actual del gasto y de la población AM se convierte en un reto para el área de mercadeo de las empresas, pero también de los gobiernos; está demostrado que éste segmento tiende a ser cada vez más voluminoso y decisivo en los mercados. Los usuarios pagan por productos Premium a cambio de calidad y beneficios para la salud, y como tal tienen un marcado crecimiento del gasto, utilizando una parte importante de su ingreso en la satisfacción de sus diversas necesidades.

A diferencia del sentir del Estado, el AM para la empresa no se observa como un problema del envejecimiento, pues debido a su importante consumo de servicios médicos, dependencia, autonomía en la toma de decisiones en la compra de productos y deseo de bienestar en general, se proyecta como una población potencial, para atender con soluciones estratégicas de mercadeo, innovación empresarial y sentido social.

Para manejar la creciente demanda de productos y servicios que solicitan los AM es preciso tratarlos de manera específica a cada necesidad, contribuyendo de forma ética y real con oferta de productos en un entorno organizacional optimo, que contribuyan para un envejecimiento que garantice la mejor calidad de vida posible. Por lo tanto, el entorno 
socioeconómico donde el AM vive hoy requiere de un enfoque más óptimo y solidario, que conduzca a la construcción de calidad de vida para éstas personas que muy bien pueden ser mentores de nuestra sociedad. Es así como, pensar en una organización solidaria más estructurada en las residencias de ésta población, para el caso colombiano, puede llegar a ser una de las formas de lograr un modelo económico y de mercado que incorpore el desarrollo social y mayor autonomía, minimizando los impactos negativos que dejan las políticas sociales en este segmento, y de esta manera rescatar esa sociedad más humana, más integradora, más optimista y más estable.

\subsection{La residencia y el adulto mayor}

La población adulto mayor, también llamada de la Tercera edad o personas mayores, según el contexto, hace referencia a los hombres y mujeres mayores de 60/65 años. Las mujeres tienen mayor esperanza de vida que los hombres en todas las regiones del mundo, esta diferencia es más pronunciada en los países desarrollados. La sociedad está envejeciendo, las personas de más 60 años son más numerosas que los menores de 14. En Estados Unidos y Japón, la esperanza de vida está por encima de los 80 años; En Colombia está situada en los 75 años. En España ${ }^{5}$ el envejecimiento de su población se debe entre otros motivos a la baja tasa de natalidad, al aumento de la esperanza de vida, y a los avances de la ciencia. Entre tanto en Colombia el inicio de la baja de la natalidad a fines de la década de los 60 puede definirse como primera fase de transición demográfica. En los próximos 50 años se espera que este proceso se profundice y llegue a completar dichos cambios. Partiendo de 1950 con una natalidad cercana a los 7 hijos por mujer, dicho indicador se redujo en un tercio hasta el año 2000, lo que significa la tasa de natalidad actual de 2.4 hijos. El descenso continuará y hacia 2050 el nivel de reemplazo se reduciría a un tercio del nivel que tuvo 100 años atrás.

Entre tanto las residencias/viviendas que acogen al AM en Colombia, se les conoce como hogar geriátrico ${ }^{6}$, también en España, hoy en día “...son considerados establecimientos residenciales para personas mayores que tienen como fin brindar servicios de alojamiento, alimentación, higiene, recreación activa o pasiva y atención médica y psicológica no sanatorial al AM de 60 años, en forma permanente o transitoria, a título oneroso o gratuito."

\footnotetext{
${ }^{5}$ La población española será la más anciana en toda la UE en el 2050. [en línea] elmundo.es. España: 2005, disponible en internet: http://www.elmundo.es/elmundo/2005/04/08/sociedad/1112964344.html

${ }^{6}$ En España esa expresión se utilizó en los siglos XVIII y XIX.

${ }^{7}$ CEDOM. Dirección general centro documental de información y archivo legislativo. sección 9: de la sanidad educación y cultura. Buenos Aires, [en línea], 2008 [citado 19 Agosto 2011] disponible en http://www.cedom.gov.ar.
} 
Este tipo de residencias se conciben dentro de los servicios sociales, que se utilizan para describir aquellas actividades organizadas, cuyo objetivo es ayudar a la adaptación mutua entre los individuos y su ambiente social. Son organizaciones que nacen y se desarrollan bajo concepciones de acogida y de servicios asistenciales, es a mediados del siglo XX cuando se incorpora el concepto de rehabilitación; a partir de la década del ochenta se orienta hacía el servicio de la salud y calidad de vida del AM. Los tipos de residencias que se conocen son: "hogar día” o "centros día” y "Hogar Residencial”.

Según lo hallado en el estudio realizado en las residencias para AM en Cali, no se tiene definida una estructura holista o formal como tal que permita tener un sistema de relaciones formales o sistémicos establecidos. Una estructura departamentalizada, matricial, híbrida, tampoco se encontró; la estructura lineal es la más cercana a lo que se conoce de los esquemas organizacionales. Todas, incluyendo las de España, se caracterizan por la estructura simple, gestionada en su mayoría, por los mismos fundadores, la contabilidad y procesos son sencillos y la autoridad está centrada en una sola persona. De aquí que la estructura organizacional en dichas residencias, en su mayoría, se configuran en sistemas rígidos, donde la pasividad, la despersonalización y el quietismo son los rasgos más destacados, claro está que también están los de sistemas flexibles, donde la actividad, la participación, el respeto por el individuo se constituye en la base de una tarea de constante cambio y ajuste al medio. Funcionan tanto en la figura pública bajo la tutela del Estado, como privada, ya sea con ánimo o sin ánimo de lucro; siendo las más predominantes en Colombia frente a España ${ }^{8}$ las fundaciones/hogar geriátrico, de tipo privado y sin la filosofía de lo que significa una empresa social, pero tampoco se visualiza asociatividad entre las mismas, como sí sucede en España con la Federación Lares.

La dirección y gestión de las residencias y centros asistenciales está abocado a brindar al AM un servicio especializado para mejorar o mantener su capacidad de atención en las diferentes áreas y así, el residente logre una mayor calidad de vida y un ambiente de hogar. Los hogares/residencias de la muestra en el estudio, cumplen con las normas establecidas en cuanto a la accesibilidad arquitectónica, para que ésta población pueda conservar su independencia y su propia autonomía, minimizando los riesgos de accidentes y aumentando

\footnotetext{
${ }^{8}$ Las residencias de personas mayores en España, sean de titularidad pública o privada -de mercado o solidaria-, responden a unos criterios preestablecidos en las normas de la administración pública, cuyas exigencias son de obligado cumplimiento, como tener una ratio determinada de personal según el número de residentes y su grado de dependencia, la profesionalidad y cualificación de las diferentes categorías del personal, etc. En definitiva, en el funcionamiento del día a día se tiene muy en cuenta los derechos y autonomía de las personas mayores residentes en dicho habita.
} 
en ellos y en sus familiares, mayor tranquilidad y seguridad. Es aquí donde la empresa de modelo social y solidario entra a jugar un papel importante; no solo con el asociado entre sí (como el caso de la Federación Lares), sino también para fortalecer sus servicios e incrementar las posibilidades de cooperación a través de cadenas productivas, desarrollando una red de organizaciones, pero que se contemple de manera integral la producción, colaboración en las compras de productos y la generación de infraestructura de apoyo y actividades de bienestar donde participe el AM con posibilidades de mayor participación e implicación social, entre otras actividades.

Un factor macroeconómico del modelo de empresa social, está en la aportación al mantenimiento del empleo en forma de emprendimiento, mientras que en el plano microeconómico radicaría en los ingresos adicionales que muy seguramente pudiesen generar dicha población mayor. Los ingresos adicionales se pueden conseguir ya sea rebajando costos y compra en colaboración, produciendo arte, escritos, manualidades, artesanías, etc. mientras su mente y cuerpo están activos, logrando así doble objetivo para el AM y la residencia. Pero además, si a éste segmento se le proyecta como mentores 'desaprovechados', que lo son en su mayoría, podrían impartir formación y comunicación con la niñez y juventud mediante redes o alianzas entre la residencia y los colegios, de esta manera los beneficios mutuos generarían bienestar a las partes. En definitiva, se trata de socializar la "inteligencia social" del AM en beneficio de la colectividad y el bien común.

\subsection{El adulto mayor. Comportamiento y bienestar en la residencia}

En la muestra de las residencias estudiadas, se destaca que se encuentra un mayor número de hombres $(65 \%)$ que de mujeres $(35 \%)$ en el caso de Colombia, esto debido a que los familiares manifiestan la preferencia de cuidar a la madre en casa, porque han sido más consagradas a su hogar, mientras que al padre lo llevan a la residencia, por lo general, por razones de irresponsabilidades, presentándose casos donde no quieren ver al padre, limitándose a pagar la mensualidad. Entre tanto, en España los AM (95\%) prefieren vivir en sus domicilios mientras puedan valerse por sí mismos en el desarrollo de sus actividades de vida diaria. A la inversa de Colombia, en España predominan las mujeres (55\%) sobre los varones (45\%); la edad de las personas que optan por vivir en centros asistenciales/residencias, rondan los 78 años, existiendo varias decenas de "centenarias". Entre las personas mayores de 80 años que cada vez aumenta más, presentan un conglomerado de polipatologías, predominando la demencia, antesala del Alzheimer, 
principalmente en varones. Pues no siempre el vivir más años, conlleva a vivir mejor y con salud.

Por otro lado y con el fin de conocer los servicios y el estado emocional del AM en la residencia, se indaga sobre las actividades que realizan y la autonomía en las compras de productos realizadas con mayor frecuencia. Se tienen en cuenta componentes que van desde la actividad física hasta las sociales -relación de afecto- y las terapéuticos como se aprecia en la tabla 1, hay diferencias significativas entre las residencias de ambos entornos analizados; mientras en la tabla 2 se visualiza el tipo de contribución o ayuda que reciben dichas residencias, encontrando que mientras en España son los centros de salud y los centros infantiles de quienes reciben colaboración en una mayor proporción, en Colombia son los colegios y otros centros; entre tanto en la tabla 3 , se destacan algunas categorías en cuanto al estado emocional y personalidad que presenta el AM residente, se subraya el entusiasmo como el más representativo.

Tabla 1. Actividades que realizan los Adultos Mayores en las Residencias

\begin{tabular}{|c|c|c|}
\hline ACTIVIDADES & \multicolumn{2}{|c|}{ Proporción } \\
& \multicolumn{1}{|c|}{ Cali } & \multicolumn{1}{|c|}{ Madrid/Avila } \\
\hline Actividades Físicas & $54,16 \%$ & $50 \%$ \\
\hline Juegos De Mesa & $50 \%$ & $35 \%$ \\
\hline Atención Afectiva Percibida & $29,16 \%$ & $45 \%$ \\
\hline Ver Televisión & $29,16 \%$ & $25 \%$ \\
\hline Terapias Ocupacionales & $25 \%$ & $40 \%$ \\
\hline Manualidades & $25 \%$ & $35 \%$ \\
\hline Escuchar Música y/o Cantar & $16,66 \%$ & $25 \%$ \\
\hline Hidroterapia & $16,66 \%$ & $10 \%$ \\
\hline Salir De Paseo & $12,5 \%$ & $35 \%$ \\
\hline Actividades Espirituales & $12,5 \%$ & $30 \%$ \\
\hline Bailar & $8,33 \%$ & $5 \%$ \\
\hline Cuidados Básicos & $8,33 \%$ & $15 \%$ \\
\hline Actividades Recreativas & $8,33 \%$ & $38 \%$ \\
\hline
\end{tabular}

Tabla 2. Instituciones que colaboran a las residencias de Adultos Mayores

\begin{tabular}{|l|c|}
\hline Categoria - MADRID/AVILA & Proporción \\
\hline Programas de psicología & $8,3 \%$ \\
\hline $\begin{array}{l}\text { Universidades con } \\
\text { prácticas en medicina }\end{array}$ & $25 \%$ \\
\hline Centros de lúdica & $33,3 \%$ \\
\hline Centros infantiles & $66,6 \%$ \\
\hline Centros de salud & $83,3 \%$ \\
\hline Otro centros de la tercera edad & $33,3 \%$ \\
\hline El Gobierno & $25 \%$ \\
\hline Fundaciones & $41,6 \%$ \\
\hline \multicolumn{1}{|c|}{ Categoría - CALI } & Proporción \\
\hline Colegios y/o escuelas & $22 \%$ \\
\hline Otros Centros de tercera edad & $22 \%$ \\
\hline $\begin{array}{l}\text { Universidades con } \\
\text { Practicantes en medicina }\end{array}$ & $17 \%$ \\
\hline Fundaciones & $9 \%$ \\
\hline Centros de lúdica & $4 \%$ \\
\hline Centros de salud & $4 \%$ \\
\hline
\end{tabular}

Tabla 3. Estado emocional de los Adultos Mayores en las Residencias

\begin{tabular}{|c|c|c|}
\hline Categoría & \multicolumn{2}{|c|}{ Cali $\begin{array}{c}\text { Proporción } \\
\text { Madrid/Ávila }\end{array}$} \\
\hline Entusiasta & $63 \%$ & $75 \%$ \\
\hline Apático & $58 \%$ & $40 \%$ \\
\hline Colérico & $50 \%$ & $35 \%$ \\
\hline Aburrido & $38 \%$ & $40 \%$ \\
\hline Hostiles & $38 \%$ & $25 \%$ \\
\hline Nervioso & $33 \%$ & $40 \%$ \\
\hline Analítico & $8 \%$ & $15 \%$ \\
\hline
\end{tabular}

Fuente: Resultados del estudio. 2013-2014 
En la investigación se quiso conocer qué compras, de tipo personal, hacían los familiares de los AM o los responsables de las residencias y si en sus preferencias tenían en cuenta la opinión de los AM, para ello se entrevistó a los encargados o responsables de las residencias/hogares geriátricos y a los familiares del AM. Los resultados fueron, de la población de 469 AM que viven en dichas residencias -caso Cali-, 378 de los familiares de estas personas compran productos y se los llevan al hogar. De acuerdo con el $45 \%$ de los encuestados, las marcas son muy importantes a la hora de realizar las compras de los productos de consumo para la residencia. Solo el 17\% de los encuestados afirmó que las marcas no tienen ninguna importancia cuando se hacen las compras. Se encuentra que la mayor proporción de los encuestados (70\%) consideran a las marcas algo o muy importantes a la hora de decidir los productos y solo el $30 \%$ considera lo opuesto. En su gran mayoría, los familiares opinan que el AM está mejor atendido en la residencia que en el hogar familiar, esto debido a los servicios que reciben en el lugar. Sin embargo en España la opinión es opuesta, casi el $90 \%$ considera que el AM se encuentra mejor en casa. En cuanto a la toma de decisión de compra, el AM de las residencias en España es más autónomo.

\section{MARCO INSTITUCIONAL DE LAS POLÍTICAS SOCIALES HACÍA EL ADULTO MAYOR EN COLOMBIA Y ESPAÑA}

La evolución de los procesos de protección y solidaridad social experimentados muy incipientemente durante los siglos XVIII y XIX, han servido de base para el análisis y la implantación de un modelo de protección que conlleve a lograr la equidad y un mejor nivel de vida de la comunidad. Ha sido durante el siglo XX donde las sociedades capitalistas optaron por establecer un modelo de intervención por parte del Estado; formulado por John Maynard Keynes, sus ideas y teorías le otorgaron un papel protagónico a la participación del Estado y permitieron prever una mejora en el bienestar de la población. La implantación de la teoría keynesiana obliga a que se establezcan políticas de desarrollo estructuralista o de oferta; buscando con esto, reformar la estructura económica de un país, permitiendo fortalecer sus instituciones. Posteriormente, la crisis de la década de los setenta, caracterizada por una aceleración de la inflación mundial y una disminución del crecimiento económico con desempleo, le crea una crisis de credibilidad al Estado interventor - benefactor, y es precisamente hacía la década de los setenta y ochenta cuando se inicia de nuevo la gran influencia de las ideas neoliberales en el mundo; la mayoría de las economías capitalistas de occidente entraron en una fase recesiva, que se enfrentó en mayor o menor medida con una 
reducción de la intervención del Estado en la economía, una flexibilización de los mercados en general (particularmente el mercado laboral) y la privatización de empresas de servicios públicos, modificándose leyes y políticas sociales que en la actualidad afectan al AM de manera negativa o en otros casos, positivamente.

En Colombia existe legislación nacional sobre las instituciones geriátricas en cuanto a reglamentar la parte higiénico-sanitaria e infraestructura física de los centros residenciales para el AM. Es así como el Protocolo Adicional de la Convención Interamericana de Derechos Humanos en materia de Derechos Económicos, Sociales y Culturales, "Protocolo de San Salvador", (1988), fue ratificado por Colombia mediante la ley 319 de 1996, la cual establece en su artículo 17 - Protección de los ancianos. El libro IV de la Ley 100 de 1993 y los decretos reglamentarios, establecen a través de un auxilio, un esquema de atención para el mejoramiento de las condiciones de vida de las personas mayores de 65 años y de 50 años en condición de discapacidad o indígenas en indefensión que se encuentran en condiciones de extrema pobreza e indigencia. Se establece la obligación para el Estado del reconocimiento de un auxilio para cubrir los costos de los servicios de salud y complementarios, así como para financiar el sistema.

Entre tanto en España, en 1963 aparece la Ley de Bases de la Seguridad Social cuyo objetivo principal era la implantación de un modelo unitario e integrado de protección social, con una base financiera de reparto, gestión pública y participación del Estado en la financiación. A pesar de esta definición de principios, muchos de los cuales se plasmaron en la Ley General de la Seguridad Social de 1966, con vigencia de 1 de enero de 1967, lo cierto es que aún pervivían antiguos sistemas de cotización alejados de los salarios reales de trabajadores, ausencia de revalorizaciones periódicas y la tendencia a la unidad no se plasmó al pervivir multitud de organismos superpuestos. La primera gran reforma se produce con la publicación del Real Decreto Ley 36/1978, de 16 de noviembre, que, en función de lo acordado en los Pactos de la Moncloa crea un sistema de participación institucional de los agentes sociales favoreciendo la transparencia y racionalización de la Seguridad Social, así como el establecimiento de un nuevo sistema de gestión.

La Entidad Gestora de la Seguridad Social, Instituto Nacional de Servicios Sociales en España, fue creada por el Real Decreto-Ley 36/1978, de 16 de noviembre, sobre gestión institucional de la Seguridad Social, la Salud y el Empleo, para la gestión de los servicios complementarios de las prestaciones del Sistema de la Seguridad Social. Hasta 1997 los 
colectivos a los que dirigía su acción el Instituto eran las personas mayores y personas con discapacidad, así como los solicitantes de asilo y refugiados, y su estructura estaba enfocada para un ámbito territorial de gestión a nivel nacional. Este modelo fue modificado por el proceso de transferencias a las Comunidades Autónomas, que prácticamente vino a culminarse a principios de 1998, de modo que en la actualidad la entidad sólo mantiene Direcciones Territoriales en las Ciudades de Ceuta y Melilla, y los centros de referencia de ámbito nacional. En efecto, el artículo 41 de la Constitución establece que los poderes públicos mantendrán un régimen público de seguridad social para todos los ciudadanos que garantice la asistencia y prestaciones sociales suficientes ante situaciones de necesidad, especialmente en caso de desempleo, indicando que la asistencia y prestaciones complementarias serán libres.

A continuación se presenta un esbozo (cuadro 1) sobre la legislación del actual sistema de seguridad social (SSS) y su cobertura al AM tanto en España como en Colombia. Conocer sobre éste tema implica adentrarnos más en las oportunidades que tiene la población de mejorar sus condiciones de vida en la adultez, como también, identificar la capacidad de poder adquisitivo de éste segmento, y lograr construir un modelo residencial social que aporte hacía su bienestar.

Cuadro 1. Síntesis de la legislación comparativa sobre Seguridad Social, EspañaColombia

\begin{tabular}{|c|c|c|}
\hline LEGISLACIÓN & ESPAÑNA & COLOMBIA \\
\hline $\begin{array}{l}\text { Básica } \\
\text { 1. Fecha de } \\
\text { implantación }\end{array}$ & $\begin{array}{l}\text { Real Decreto de } 11 \text { de marzo de } 1919 . \\
1963 \text { Ley de Bases de la Seguridad Social }\end{array}$ & $\begin{array}{l}\text { Ley } 29 \text {, de } 1905 . \text { Ley 53, de } 1945 . \\
\text { Ley } 77 \text {, de 1959. Decreto } 3.041 \text {, de } 19 \\
\text { Diciembre de } 1966 .\end{array}$ \\
\hline $\begin{array}{l}\text { 2. Normas } \\
\text { Básicas } \\
\text { Vigentes }\end{array}$ & $\begin{array}{l}\text { Texto Refundido de la Ley General de S.S. } \\
\text { (Real Decreto Legislativo } 1 / 94 \text {, de } 20 \text { de } \\
\text { junio. Decreto } N^{\circ} 3.158 \text {, Dic. } 23 \text { de } 1966 \text { ). }\end{array}$ & $\begin{array}{l}\text { Ley 100, de 1993, y Ley } 797 \text { de } 2003 \text { y sus } \\
\text { Decretos reglamentarios. }\end{array}$ \\
\hline $\begin{array}{l}\text { 3. Aplicación } \\
\text { Personal. Personas } \\
\text { protegidas }\end{array}$ & $\begin{array}{l}\text { Trabajadores por cuenta ajena y propia. } \\
\text { Pensiones no contributivas: residentes que } \\
\text { carezcan de recursos, en la actual legislación. }\end{array}$ & $\begin{array}{l}\text { Trabajadores vinculados con contrato de } \\
\text { trabajo o como servidores públicos y } \\
\text { trabajadores independientes }\end{array}$ \\
\hline $\begin{array}{l}\text { 4. Requisitos } \\
\text { básicos de acceso a } \\
\text { la prestación }\end{array}$ & $\begin{array}{l}\text { Pensiones contributivas: Período mínimo de } \\
15 \text { años, de los cuales } 2 \text { deben estar } \\
\text { comprendidos dentro de los } 10 \text { años } \\
\text { inmediatamente anteriores al hecho causante. } \\
\text { Cumplidos } 65 \text { años (para hombre y mujer). } \\
\text { Cesar en el trabajo. } \\
\text { Pensiones no contributivas: Carecer de } \\
\text { ingresos suficientes. Cumplidos } 65 \text { años. } \\
\text { Residencia en España. }\end{array}$ & $\begin{array}{l}\text { Régimen de Prima Media: Haber cotizado } \\
\text { un mínimo de } 1100 \text { semanas. } 57 \text { años } \\
\text { mujer y } 62 \text { hombre. } \\
\text { Régimen de Ahorro individual: No se } \\
\text { exige edad mínima (poseer una cuenta con } \\
110 \% \text { del salario mínimo mes legal vigente } \\
\text { al año } 1993 \text {. Existen reglas específicas en } \\
\text { la prestación con cargo al Fondo de } \\
\text { Garantía de Pensión Mínima. }\end{array}$ \\
\hline $\begin{array}{l}\text { 5. Nivel } \\
\text { cuantitativo } \\
\text { de la prestación }\end{array}$ & 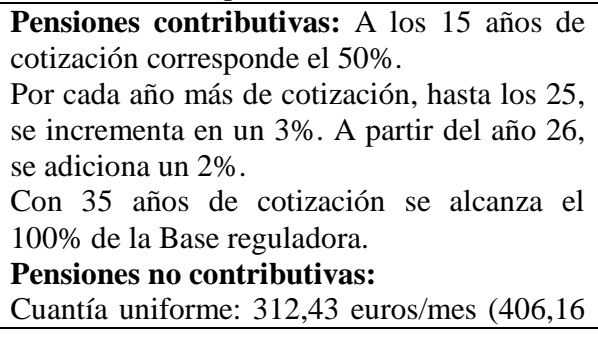 & $\begin{array}{l}\text { Régimen de Prima Media: } 65 \% \text {, del } \\
\text { ingreso base de liquidación de los afiliados. } \\
\text { Desde el 2005, por cada 50 semanas } \\
\text { adicionales a las mínimas requeridas, el } \% \\
\text { se incrementa en un } 1,5 \% \text { del ingreso base } \\
\text { de liquidación, llegando a un monto } \\
\text { máximo de pensión del } 80 \% \text { del ingreso. } \\
\text { El valor total de la pensión no podrá ser } \\
\text { superior al } 80 \% \text { del ingreso base de }\end{array}$ \\
\hline
\end{tabular}




\begin{tabular}{|c|c|c|}
\hline & US\$) $(1$ euro $=1,23$ US\$). & liquidación, ni inferior a la pensión mínima \\
\hline $\begin{array}{l}\text { 6. Base } \\
\text { reguladora } \\
\text { de la prestación }\end{array}$ & 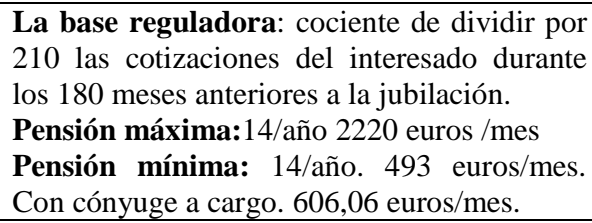 & $\begin{array}{l}\text { El Ingreso base de liquidación es el } \\
\text { promedio de los salarios sobre los cuales ha } \\
\text { cotizado el afiliado durante los } 10 \text { años } \\
\text { anteriores a la pensión o el promedio de } \\
\text { toda la vida, ajustado por la inflación. De } \\
\text { debe haber cotizado más de } 1250 \text { semanas }\end{array}$ \\
\hline 7. Actualización & $\begin{array}{l}\text { Actualización automática al principio de cada } \\
\text { año, de acuerdo con el Índice de Precios al } \\
\text { Consumo previsto para el año del que se } \\
\text { trate. }\end{array}$ & $\begin{array}{l}\text { Las pensiones se actualizan cada } 10^{\circ} \text { de } \\
\text { enero, en función de la variación IPC. Las } \\
\text { iguales al salario mínimo se actualizan con } \\
\text { el incremento del salario mínimo o del IPC. }\end{array}$ \\
\hline $\begin{array}{l}\text { 8. Afinidad } \\
\text { con el trabajo }\end{array}$ & $\begin{array}{l}\text { La pensión se suspende en el caso de que se } \\
\text { siga ejerciendo una actividad profesional } \\
\text { retribuida. }\end{array}$ & $\begin{array}{l}\text { La pensión de vejez es compatible con } \\
\text { cualquier actividad retribuida, pero no } \\
\text { podrá cotizarse para una nueva pensión. }\end{array}$ \\
\hline $\begin{array}{l}\text { 9. Imposiciones } \\
\text { fiscales }\end{array}$ & $\begin{array}{l}\text { Prestaciones sujetas a impuestos en su } \\
\text { totalidad. }\end{array}$ & $\begin{array}{l}\text { Las pensiones están gravadas donde } \\
\text { excedan de } 25 \text { SMLV (US } \$ 5.421,25) \text {. }\end{array}$ \\
\hline
\end{tabular}

Fuente: Banco de Información de los Sistemas de Seguridad Social Iberoamericanos - 2012 (BISSI 2012). Tesis de grado: Salcedo J. L. Dirección: Osorio L. "Una perspectiva de la realidad social y el actual panorama de las políticas gubernamentales y sociales de la población de la tercera edad en Colombia" Universidad Autónoma de Occidente. 2014.

\section{LA PROTECCIÓN SOCIAL A PERSONAS MAYORES. UN ANÁLISIS PARA COLOMBIA Y ESPAÑA}

En Colombia, el S.S.S. fue instituido con la Ley 100 de 1993, que tiene por objeto garantizar los derechos irrenunciables de la persona y de la comunidad para garantizar la calidad de vida de la población; se divide en 4 sesiones, el primer libro está dirigido al sistema general de pensiones y el $4^{\circ}$ a los Servicios Sociales complementarios. Con la puesta en marcha de ésta Ley se da un cambio trascendental a la administración y prestación del servicio en Colombia. Con el fin de brindar mayor y mejor eficiencia al sistema, se estableció que instituciones tanto públicas como fondos privados pudieran administrar y prestar el servicio de salud. De acuerdo con su función y alcance, éstas instituciones se clasifican en: entidades territoriales de salud, entidades administradoras de planes y beneficios o (EAPB), las Entidades Promotoras de Salud (EPS), las Administradoras de Riesgos Profesionales (ARP), Instituciones Prestadoras de Servicio (IPS) y Empresas Sociales del Estado (ESE).

La historia en Colombia se remonta a finales del Siglo XIX en salubridad pública y asistencia pública; pero es con la creación de la Caja de Previsión Nacional en el año 1945 cuando se crea el Sistema de Seguridad Social - SSS -. Otro aspecto importante que marca los indicios del SSS es la creación de las Cajas de Compensación Familiar en 1950. Es en 1946 cuando se crea el Instituto Colombiano de Seguros Sociales (ISS), entidad del Estado que cubrió hasta el 2007 al trabajador privado con empleo formal. En 1990 se inicia el proceso de liquidación del ISS, previo a la nueva Constitución política de Colombia (1991) donde se define la Seguridad Social como un servicio público permanente y un derecho colectivo, consagrando sus principios rectores, la gestión privada y descentralizada por niveles de 
atención. Pero es entre el 2007 y el 2008 cuando se privatizan sus entidades (IPS, ARP y EPS), en el 2012 deja de existir definitivamente este Instituto, quedando Colpensiones a cargo de las Pensiones de los trabajadores en el Régimen de Prima Media. Este SSS estuvo marcado notablemente por la diferencia entre el Sistema que cubría a trabajadores privados (Seguridad Social) y funcionarios públicos (Previsión Social).

Las políticas de protección social en España se inician con la Comisión de Reformas Sociales (1883) que se encargó del estudio de cuestiones que interesasen a la mejora y bienestar de la clase obrera. En 1900 se crea el primer seguro social, la Ley de Accidentes de Trabajo, y en 1908 aparece el Instituto Nacional de Previsión, en el que se integran las cajas que gestionan los seguros sociales que van surgiendo.

En España, el IMSERSO, como Entidad Gestora de la Seguridad Social (S.S.) adscrita al Ministerio de Sanidad, Servicios Sociales e Igualdad a través de la Secretaría de Estado de Servicios Sociales e Igualdad, con naturaleza de entidad de derecho público y capacidad jurídica para el cumplimiento de los fines que le están encomendados a tenor del artículo 59.1 del texto refundido de la Ley General de la Seguridad Social, aprobado por el Real Decreto Legislativo 1/1994, de 20 de junio ${ }^{9}$,tiene competencias en las siguientes materias:

- La gestión y seguimiento de las pensiones de invalidez y jubilación en sus modalidades no contributivas y en la forma prevista en la disposición adicional.

- Los servicios complementarios de las prestaciones del S. S. Social.

- El seguimiento de la gestión de las prestaciones económicas derivadas de la Ley 13/1982, de integración social de las personas con discapacidad.

- La propuesta normativa básica que garantice los principios de igualdad y solidaridad para la determinación de los baremos, a los efectos del reconocimiento del grado de discapacidad.

- La propuesta y ejecución, en su caso, de las funciones atribuidas al Instituto de Mayores y S. S. artículo 9.1.a del Real Decreto 1600/2004, en concreto "La planificación y regulación básica del reconocimiento del derecho a una ayuda personalizada a toda persona dependiente, garantizando un sistema de servicios universal, integrado y uniforme".

\footnotetext{
${ }^{9}$ Consultado en Real Decreto-ley 36/1978, de 16 de noviembre, sobre gestión institucional de la Seguridad Social, la salud y el empleo. Boletín Oficial del Estado. Gobierno de España. Página WEB: http://www.boe.es/buscar/doc.php?id=BOE-A-1978-28739.
} 
- El fomento de la cooperación con las organizaciones y entidades que agrupan a las personas mayores.

- El establecimiento y gestión de centros de atención especializada o de aquellos a los que se les asignen objetivos especiales de investigación de ámbito de actuación estatal en el campo de acción del Instituto.

- La propuesta, gestión y seguimiento de planes de servicios sociales de ámbito estatal en las áreas de personas mayores y de personas dependientes.

\section{LA ECONOMÍA SOCIAL EN LOS SERVICIOS DE BIENESTAR SOCIAL}

La relación entre la población AM, las políticas sociales, las residencias para éste segmento y la calidad de vida, atañe directamente al modelo de economía social. La solidaridad entre los seres humanos ha sido una condición de vida en sociedad, durante toda la historia de la humanidad, las personas han respondido a su instinto gregario, permitiendo formar comunidades. La Economía Social en los últimos años ha experimentado un considerable crecimiento en el sector de los servicios sociales, buscando el bienestar y la calidad de vida de la población, y lo hace en clara competencia con las empresas privadas. Esta realidad les ha exigido adoptar formas de organización cada vez más estructuradas y eficientes, convirtiéndose en referente de los sistemas de bienestar (salud, protección social y servicios asistenciales), a través de entidades que son cada vez más empresariales y que dirigen su oferta a sectores vulnerables de la sociedad y el mercado libre, superando el nicho de exclusión social que ocupaban tradicionalmente (Etxezarreta y Bakaikoa, 2011, 35-36).

Es así como las empresas sociales como las cooperativas han jugado un importante papel en la construcción y desarrollo del Sistema de Servicios Sociales. Como se ha señalado en un estudio (COCETA, 2011, 13), en los años 80 las necesidades de contención del déficit público para alcanzar la convergencia nominal con Europa convivían, de forma contradictoria, con las necesidades de consolidar redes de protección social básicas que permitieran avanzar en la convergencia real. Para dar respuesta a las necesidades sociales que se estaban planteando, las entidades de iniciativa social, desarrollaron proyectos novedosos en áreas en las que el Estado todavía no había llegado ni asumido su responsabilidad.

La sociedad civil, a través de la iniciativa social, ha ido asumiendo el papel en la provisión de servicios de bienestar, no limitándose a cubrir los huecos que la acción pública y el mercado no atienden o han abandonado. Más allá de la visión subsidiaria o sustitutiva, se 
ha abierto paso el convencimiento de que su contribución es especialmente adecuada y válida para contribuir a la mejora del bienestar, y que constituye un medio idóneo para promover los valores de solidaridad, para situar a la persona como centro y objetivo de la acción y para establecer mecanismos de control del Estado y del Mercado por parte de los ciudadanos (COCETA, 2011, 14). La sociedad civil debe asumir su papel, alternativo y complementario, de catalizador, motor y tractor del cambio (Salinas, Osorio y Albarrán, 2011, 15).

Las alianzas estratégicas merecen destacarse como uno de los factores clave del desarrollo del sector cooperativo de servicios a las personas, así como la integración e intercooperación para adquirir el tamaño, los recursos técnicos y la capacidad de inversión suficiente para sobredimensionarse en un contexto competitivo y lograr la sostenibilidad a medio largo plazo. Algunas buenas prácticas confirman lo que se acaba de decir (Jiménez y Rodríguez, 2012: 32-34).

Claros, Sociedad Cooperativa Andaluza, una de las cooperativas más importantes del país, es el resultado de la fusión de una serie de iniciativas cooperativas pequeñas, dispersas geográficamente y probablemente abocadas a la desaparición, que a través de una dinámica participativa y autogestionada han logrado convertirse en una organización eficiente, que genera empleo y cuenta con un gran impacto sectorial y un alcance territorial considerable (Morales Gutiérrez, 2011, 221-223). La cooperativa de integración denominada Transformaciones Sociosanitarias Andaluzas, TRASSA, dedicada a la construcción y gestión de servicios sociosanitarios y socioeducativos, de la que forman parte importantes grupos empresariales cooperativos, como Sierra Nevada (Granada) o Macrosad(Jaén).

La constitución en 2005 de la primera cooperativa andaluza de tercer grado, Novocare, de la que forman parte TRASSA, el Grupo sociosanitarioYissi, y las cooperativas de segundo grado de construcción CONSUR y Tilodissa, entre otras, cuya principal actividad es la atención sociosanitaria. La creación en Córdoba, en 2009, de la cooperativa de integración Servicios Integrales Sociosanitarios, formado por cinco cooperativas, tres sociedades limitadas y una sociedad laboral, que realizan su actividad en una veintena de municipios de las provincias de Córdoba, Málaga, Jaén y Toledo (Morales Gutiérrez, 2011, 218-219). La segunda empresa cooperativa sociosanitaria española por volumen de facturación y empleo es Suara Cooperativa, nacida en 2008 como resultado de la fusión de tres cooperativas catalanas que desarrollaban actividades diferenciadas y complementarias CTF ServeisSociosanitaris (Barcelona, atención domiciliaria), EAS (Barcelona, atención a personas en riesgo de 
exclusión) y Escaler Cooperativa (La Garrotxa, atención a la infancia, juventud, familias, personas mayores, actividades ocupacionales e inserción laboral). El resultado de esta fusión ha sido una cooperativa con capacidad para ofrecer un amplio abanico de servicios (centros residenciales, diurnos y nocturnos, asistencia domiciliaria, escuelas y centros educativos, servicios de orientación, preventivos y de apoyo) que atiende a más de 16.000 usuarios y gestiona 112 centros en 15 comarcas catalanas. Suara se ha integrado en el grupo Clade, el primer grupo cooperativo multisectorial catalán (más de 4.100 empleos y unos resultados consolidados de 305 millones de euros en 2010).

El grupo cooperativo SSI (Servicios Sociales Integrados), que gestiona varios servicios de ayuda a domicilio en Vizcaya, centros de convalecencia, unidades de media estancia, servicio de urgencias sociales y servicios de acompañamiento. La cooperativa GSR (Gestión de Servicios Residenciales), vinculada a la Corporación Mondragón, que gestiona ocho residencias, varios centros de día, viviendas comunitarias y diversos servicios de ayuda a domicilio. Las residencias de mayores y los centros de día de la cooperativa Tosande, del Grupo Gredos San Diego, que figuran como unos de los mejor valorados de la zona sur de Madrid.

La Economía social es una realidad y está siendo reconocida en diversas instancias políticas, sociales y económicas. La Ley de la Dependencia no se refiere directamente a la economía social, sí lo hace indirectamente definiendo al Tercer Sector como "organizaciones de carácter privado surgidas de la iniciativa ciudadana o social, bajo diferentes modalidades que responden a criterios de solidaridad, con fines de interés general y ausencia de ánimo de lucro, que impulsan el reconocimiento y el ejercicio de los derechos sociales" (artículo 2.8). Un claro ejemplo de esta diversidad es el caso del tercer sector de acción social que interviene en la atención a la exclusión social y a las diversas formas de dependencia en España, donde junto a las asociaciones voluntarias que prestan servicios en ámbitos a los que no llega la intervención pública, como por ejemplo en las zonas rurales, opera también una red de fundaciones aglutinada mayoritariamente en torno a la Federación Lares, vinculada a entidades religiosas, que prestan servicios de responsabilidad pública, y un amplio número de cooperativas, que han entrado con fuerza en la provisión de servicios de atención a la dependencia (Etxezarreta y Bakaikoa, 2011, 42).

Cabe destacar como parte del trabajo realizado, la muestra de residencias españolas, hacen parte de la Federación de Residencias y Servicios de atención a los A.M., sector 
solidario, Lares ${ }^{10}$, organización que se inspira en los valores del humanismo cristiano, apuesta por la permanente mejora, por la transparencia y por un enfoque solidario de su gestión, donde la persona es el centro de su quehacer. A Lares Federación pertenecen 17 Asociaciones autonómicas que agrupa a un total de 970 empresas (centros y servicios) donde viven y reciben cuidados alrededor de 70.000 mayores y son atendidos por 35.000 trabajadores profesionales de diversas ramas de la salud y servicios sociales, además cuenta con el apoyo de 9.500 voluntarios. Lares tiene una opción preferente por los AM más vulnerables y en riesgo de exclusión, presta sus servicios en zonas rurales. Lares representa al sector social de atención a los mayores ante las administraciones, las empresas, los sindicatos del sector y los medios de comunicación, defendiendo los intereses comunes.

"En Andalucía", según datos del Directorio Central de Empresas del INE, existen 1.406 empresas correspondientes a asistencia en establecimientos residenciales y actividades de servicios sociales sin alojamiento. De ellas, 236 pertenecen al sector de la Economía Social (173 cooperativas y 63 sociedades laborales), lo que supone un 17\% del total andaluz y un $3,2 \%$ del entramado empresarial de la economía social andaluza. El sector sociosanitario en la economía social da empleo a 7.479 personas (aproximadamente 6.500 en cooperativas), lo que supone un 11,1\% del empleo que genera la economía social andaluza. Podemos observar cómo un 3,2 \% de las empresas generan un 11,1 \% de empleo, por tanto son más intensivas en personal que otros sectores. Las cooperativas sociosanitarias, según datos estimados, cuentan con 2.150 plazas residenciales, 1.000 plazas de unidades de estancia diurna y presta servicios de ayuda a domicilio a 14.830 personas. Dentro del programa de Envejecimiento Activo, las cooperativas sociosanitarias atienden a 1.900 personas. Se estima que el total de personas atendidas por estas cooperativas alcanza los 20.000 usuarios" (FAECTA, 2013).

En un estudio de CEPES $^{12}$ se confirma que la presencia de la Economía social en las ramas de actividad de servicios sociales en España es alta en términos relativos, ya que representa en torno a un $10 \%$ de la oferta (de cada 100 empresas de este sector de servicios sociales para A.M. y personas con discapacidad, 10 son empresas de Economía Social) mientras que su peso en el total de la economía es del 3,9\%. Esta presencia de la Economía Social es particularmente significativa $(16,1 \%)$ en el caso de los servicios sociales sin

\footnotetext{
${ }^{10}$ Información recogida de la Memoria Institucional 2013. Lares Federación y Asociaciones surge para agrupar a los centros y servicios a los mayores sin ánimo de lucro. interlocutores ante los gobiernos, central, autonómico y local.

${ }^{11}$ Datos de la Asamblea sectorial de las Cooperativas sociosanitarias FAECTA, Granada 8 de marzo 2013.

${ }^{12}$ Estudio realizado por CEPES (2011) con la colaboración de Abay Analistas Económicos y Sociales (Martínez Marín, $\mathrm{M}^{\mathrm{a}}$ Isabel, directora) a partir de la Muestra Continua de Vidas Labores.
} 
alojamiento tanto para personas mayores como para personas con discapacidad. La especialización productiva de la Economía Social en servicios ligados a la dependencia y a la atención de A.M. y con discapacidad es, como se desprende de estos datos, muy significativa.

En cuanto al entorno de la economía Social/Solidaria en Colombia, con especial referencia a los servicios sociosanitarios, a diciembre de 2012 (Confecoop, 2013: 30) se estimó en 8.473 entidades, distribuida en los tres subsectores que lo conforman (tabla 4), corresponden 6.421 a cooperativas (75,8\%), 1.821 a fondos de empleados $(21,5 \%)$ y 231 a asociaciones mutuales (2,7\%). Las personas asociadas ascienden a 6.681 .877 de las cuales el 82,9\% (5.541.080 personas) estuvieron vinculadas a cooperativas, el 14,5\% (965.784 a fondos de empleados y el 2,6\% (175.013) a asociaciones mutuales. El empleo generado por este sector de la economía, en 2012, fue de 522.614 puestos de trabajo, tanto bajo el régimen laboral tradicional como por medio de las cooperativas de trabajo asociado. La casi totalidad del empleo fue generado por las cooperativas 512.834 empleados $(98,1 \%)$, los fondos de empleados, 5.650 empleados $(1,1 \%)$ y las mutuales, 4.130 empleados $(0,8 \%)$.

Tabla 4. La Economía Solidaria en cifras, Colombia, 2012

\begin{tabular}{|l|c|c|c|c|c|c|}
\hline \multicolumn{1}{|c|}{ Subsector } & $\mathbf{N}^{\mathbf{0}}$ Entidad & \% & Socios & \% & Empleo & \% \\
\hline Cooperativas & 6.421 & 75,8 & 5.541 .080 & 82.9 & 512.834 & 98,1 \\
\hline Fondos de Empelados & 1.821 & 21,5 & 965.784 & 14,5 & 5.650 & 1,1 \\
\hline Mutuales & 231 & 2,7 & 175.013 & 2,6 & 4.130 & 0,8 \\
\hline TOTAL & 8.473 & 100 & 6.681 .877 & 100 & 522.614 & 100 \\
\hline
\end{tabular}

Fuente: elaboración propia, basado en datos de Confecoop, 2013.

La empresa solidaria, en 2012, estuvo presente en los departamentos de Bogotá, D.C., Valle del Cauca, Antioquia y Santander, concentrándose en éstos el 53,5\% de las entidades, $77,3 \%$ de los activos del sector cooperativo nacional, 79,8\% de los pasivos, $73,4 \%$ del patrimonio, 64,3\% de los ingresos, 67,0\% de los excedentes, 75,9\% de los asociados y 72,1\% de los empleados" (Confecoop, 2013: 30).

Las personas asociadas al cooperativismo suponen el 11,9\% de la población total del país en 2012, ascendiendo a $16.623 .240(35,7 \%)$ los beneficiarios directos de la actividad de este subsector de la población de Colombia. Es decir que algo más de uno de cada tres colombianos están relacionados directamente con la economía solidaria. El empleo generado por las cooperativas, como media, representan el 2,2\% de la población económicamente activa y el 2,5\% de la población ocupada. La distribución por subsectores de actividad, son 3 las actividades que concentran el $83,1 \%$ de los puestos de trabajo generados: otras actividades, 48,9\%; servicios sociales y de salud, 20,8\% de intermediación financiera, 13,4\%. 


\section{CONCLUSIONES}

Es manifiestamente claro que en el caso colombiano hay un gran trecho aún por recorrer en el contexto de los servicios asistenciales, entre ellos los centros residenciales para el AM respecto a la experticia española como referente. Se analizaron las actividades que éstos realizan en las residencias para lograr un estado emocional positivo, los servicios que reciben y esperan recibir, las redes de colaboración, los productos que consumen y hasta la importancia sobre las marcas de lo que consumen entre otros aspectos. Son claras las disparidades entre ambos contextos analizados, tal es el caso de la autonomía en la decisión de compra y la decisión de estar o no en la residencia, además de la estructura organizacional asociativa. En Colombia respecto a España, aún es más individualista el entorno, se encuentra en crecimiento y son más de carácter privado; los familiares del residente y el mismo AM no están en la toma de decisiones sobre el consumo. Por tal razón, se debe depurar las ofertas de productos y servicios, además de analizar el componente social para el caso de Colombia.

Para ambos países, la protección de los derechos al AM se ha convertido en una política de Estado. cuentan con una "Política Nacional de Envejecimiento y Vejez", pero definitivamente el AM en España es mucho más independiente, por la facilidad de desplazamiento y el amplio portafolio de servicios ofrecido desde la iniciativa pública, privada y social; lo que conduce que a través de la Federación Lares y la integración de cooperativas exista un conocimiento y comunicación entre las residencias de las diferentes iniciativas. Por esta razón nos atrevemos a manifestar que la empresa y estructura del modelo de economía social, juega un papel preponderante para mejorar la calidad de vida del AM y en la construcción y desarrollo del sistema de servicios socio-asistenciales, teniendo presente que las políticas públicas orientadas el bienestar social están consolidadas para ambos países. Entre tanto, es necesario tener presente que las alianzas estratégicas son un factor clave del desarrollo del sector cooperativo de servicios a las personas, de integración e intercooperación, de ahí, la alta presencia de la economía social en las ramas de actividad de servicios sociales en España especialmente.

Las residencias en la ciudad de Cali, donde se aplicó el estudio inicial, no tienen definido una estructura establecida ni un plan estratégico que permita tener clara las funciones administrativas y los objetivos trazados. El AM no muestra deseos de consumir algún producto o marca específica debido a que la mayoría no se les facilita la toma de decisiones sobre el consumo o la compra, puesto que sufren problemas mentales. El proceso 
de toma de decisiones identificado arroja que directivos o administradores o los mismos familiares son los que deciden el producto o marca a comprar. También es necesario tener presente la tipología o figura de Centro con un componente más social para el caso de Colombia, puesto que en la actualidad predominan los hogares de tipo privado, o en su defecto, fundaciones en el entorno de la economía social, mientras que España cuenta con la Federación de Residencias Lares y otras asociaciones y federaciones que agrupan a los centros residenciales prestándoles servicios e interlocución ante los gobiernos -autonómico o estatal- en todo lo relacionado con el AM.

Se encuentra que el entorno de las residencias hace que el AM se sienta más seguro de sí mismo, por la atención personalizada que se le ofrece. Los familiares también influyen en su comportamiento, dado que al AM le motiva la visita de sus seres queridos, que ayudan a que su estado de ánimo se mantenga positivo y con alto grado de estabilidad psíquica y física. Sin embargo, es opuesto en los contextos analizados, mientras en uno se siente el abandono, en otro se percibe la preocupación del familiar hacía el residente. El AM residente y sus familiares están muy satisfechos con la residencia y valoran muy positivamente los diversos servicios que reciben. En España se percibe que es mayor el número de personas mayores que deciden por cuenta propia ir a vivir en una residencia, cosa que aún no sucede en Colombia.

La cartera servicios y alternativas que se ofrece al AM, en España, es amplia, entre otros son: centros residenciales en sus diferentes modalidades, de estancias diurnas y nocturnas, centros sociales diurnos, servicios de ayuda a domicilio y de teleasistencia. Estas prestaciones son ofertadas desde iniciativa pública, privada y social. A pesar de existir una oferta muy amplia especialmente de la privada, no hay suficientes servicios para que todos puedan acceder a ellos.

Actualmente ambos países cuentan con políticas de envejecimiento y vejez dirigida a toda la población, en especial, a los mayores de 60 años. Principalmente aquellos que se encuentran en condiciones de vulnerabilidad social, económica y de género. Esta es una política pública, de Estado, con el propósito de visibilizar e intervenir la situación para el AM. En los últimos años la protección de los derechos a los AM se ha convertido en una política de Estado, esto debido al creciente registro de vulneración de los derechos de los mismos.

Las políticas públicas orientadas al bienestar social están consolidadas en ambos países, aunque en los últimos años como consecuencias de la crisis económica y financiera las 
prestaciones sociales han sufrido restricciones, recortes, retrasos en pagar y cobrar, en algún momento y en determinadas situaciones la calidad del servicio se ha resentido. Son las empresas de la Economía Social las que mejor han soportado las consecuencias de las crisis económicas, no han disminuido sus servicios ni mucho menos la calidad. El bienestar social que el conjunto de la ciudadanía venía disfrutando se ha resentido, requiere una reforma profunda, una recreación del Estado de bienestar ajustado a los nuevos tiempos y necesidades con la participación y compromiso de las instituciones públicas y de la ciudadanía. Se requiere un pacto social por el bienestar del conjunto de la ciudadanía.

\section{BIBLIOGRAFÍA}

ABELLÁN GARCÍA, A., VILCHES FUENTES, J. y PUJOL RODRÍGUEZ, R. (2013) Un perfil de las personas mayores en España, 2014. Indicadores estadísticos básicos. Madrid, Informes Envejecimiento en red $n^{\circ}$ 6. [Fecha de publicación: 14/02/2014, en red 12 de marzo, http://envejecimiento.csic.es/documentos/documentos/.

ALIANZA COOPERATIVA INTERNACIONAL -ACI- (2013) Plan para una década cooperativa. 44 pp. Disponible en www.ica.org y en www.monitoringris.org.

BELTRAN, M. (1985) Cinco vías de acceso a la realidad social. Revista Española de Investigaciones Sociológicas, № 29, pp. 7-41.

CONFEDERACIÓN ESPAÑOLA DE COOPERATIVAS DE TRABAJO ASOCIADO, COCETA (2011) Cuando se trata de personas, las cooperativas llevan la iniciativa. Cooperativas de trabajo de iniciativa social. Radiografía de un sector en evolución. Madrid: Coceta, 135 pp.

CONFEDERACIÓN DE COOPERATIVAS DE COLOMBIA, CONFECOOP (2013)

Desempeño Sector Cooperativo Colombiano 2012. Un años para la historia ... y el comienzo de la década cooperativa. Disponible en www. confecoop.coop última visita diciembre 2014.

DÍAZ FONCEA, M. y MARCUELlO SERVÓS, C. (2009) La Economía Social desde el cooperativismo. Cuadernos del Mercado de Trabajo, No 3, pp. 33-42.

DÍAZ, R. (coord.) (2009) Las personas mayores en España. Datos estadísticos estatales y por Comunidades Autónomas. Informe 2008, Tomo I. Madrid. Ministerio de Educación, Política Social y Deporte. Instituto de Mayores y Servicios Sociales, (IMSERSO), 476 pp. DOYAL, L. y GOUGH, I. (1994) Teoría de las necesidades humanas. Barcelona. IcariaFuhem. 280 pp. 
EQUIPO PORTAL MAYORES (2010) Estadísticas sobre residencias: distribución de centros y plazas residenciales por provincia. Informes Portal de Mayores, $\mathrm{N}^{\circ} 104.19$ pp. ISSN 18856780.

ESPINOSA, E. y LIBREROS, M. (1995) Modelo básico para el mejoramiento de la calidad de vida del viejo recluso durante su permanencia en la cárcel del distrito judicial de Cali. Tesis de Grado. Santiago de Cali: Universidad de San Buenaventura, 125 pp.

ETXEZARRETA ETXARRI, E. y BAKAIKOA AZURMENDI, B. (2011) La participación de la Economía Social en el ámbito de la dependencia: una aproximación teórica. CIRIECEspaña, Revista de Economía Pública, Social y Cooperativa, № 71, pp. 25-48.

FEDERACIÓN DE RESIDENCIAS Y SERVICIOS DE ATENCIÓN A LOS A.M., SECTOR SOLIDARIO, Lares (2014) Memoria Institucional 2013.

FEDERACIÓN DE COOPERATIVAS DE TRABAJO EN ANDALUCÍA, FAECTA (2013) Datos de la Asamblea sectorial de las Cooperativas sociosanitarias FAECTA, Granada 8 de marzo 2013. Disponible http://www.faecta.coop.

FERICGLA, J. M. (1992) Envejecer. Una antropología de la ancianidad. Barcelona: Anthropos, 448 pp.

FERNÁNDEZ, R. (1992) Mitos y realidades sobre la vejez y la salud. Barcelona: SG Editores y Fundación Caja Madrid, 180 pp.

FERNÁNDEZ-BALLESTEROS, R. ZAMARRÓN, M. y MACÍA, A. (1996) Calidad de Vida en la Vejez en los distintos contextos. Madrid: Ministerio de Trabajo y Asuntos Sociales. Instituto Nacional de Servicios Sociales (INSERSO), 340 pp.

GARCÍA ALONSO, J. Vidal (2008) La Ley de Dependencia y las empresas de participación de trabajo asociado de la Ciudad de Madrid. En: GARCÍA-GUTIÉRREZ FERNÁNDEZ, C. y LEJARRIAGA PÉREZ DE LAS VACAS, G. (dir.): Libro blanco de la Economía Social de la ciudad de Madrid (Nuevos retos y nuevas oportunidades para las empresas de participación de trabajo). Madrid: Escuela de Estudios Cooperativos. pp. 285-320.

GÓMEZ GARCÍA, Jesús M. (2008) Economía social y atención a la dependencia: efectos económicos y repercusiones sobre el empleo. Revista Universitaria de Ciencias del Trabajo, Vol. 9/2008, pp. 227-251.

JIMÉNEZ LARA, A. y RODRÍGUEZ CASTEDO, A. (2012) La Economía Social y la atención a la dependencia. Propuestas para contribuir al desarrollo de los servicios de atención de la dependencia y a la generación de empleo estable y de calidad. No. 177. Madrid: Fundación Alternativas, 180 pp. 
MARTÍNEZ MARÍN, I. (Dir.) (2011) El impacto socioeconómico de las entidades de Economía Social. Identificación, medición y valoración de los efectos vinculados a los principios de actuación de las empresas de la Economía Social. Madrid: Confederación Empresarial Española de la Economía Social (CEPES). 114 pp. Disponible en http://www.cepes.es.

MORALES GUTIÉRREZ, A.C. (2011) Modelos de integración y desarrollo estratégico de las cooperativas sociosanitarias andaluzas. CIRIEC-España, Revista de Economía Pública, Social y Cooperativa, $\mathrm{N}^{\mathrm{o}}$ 71, pp. 203-226.

ORGANIZACIÓN NACIONES UNIDAS (2012). Informe de la Segunda Asamblea Mundial sobre el Envejecimiento. Disponible en: http://www.monitoringris.org/documents/norm_glob/mipaa_spanish.pdf.

OSORIO B. L.; SALINAS R. F.; BELTRAN Á.; PALMA M. L. (2014) Variables de Influencia en la Preferencia y Lealtad de Marca, en Residencias de Adultos Mayores. Un Análisis Social Comparativo Entre Ávila-Madrid (España) y Santiago de Cali (Colombia). Informe de la investigación. 350 pp.

SALINAS, F. (coord.), (2011) El papel de la economía social en la aplicación de la Ley de Dependencia. CIRIEC-España, Revista de Economía Pública, Social y Cooperativa, $\mathrm{N}^{\mathrm{o}}$ 71 , agosto, pp. 5 - 8.

SALINAS, F, OSORIO, L. E. y ALBARRÁN, C. (2011) El sector público y la Economía Social en la atención a la dependencia, CIRIEC-España, Revista de Economía Pública, Social y Cooperativa, No 71, agosto, pp. 9 - 24.

SALINAS RAMOS, F., OSORIO BAYTER, L.E. y ALBARRÁN FERNÁNDEZ, C. (2011) El sector público y la Economía Social en la atención a la dependencia. CIRIEC-España, Revista de Economía Pública, Social y Cooperativa, No 71, pp. 9-24.

SALCEDO J. L. (2014) Una Perspectiva de la Realidad Social y el Actual Panorama de las Políticas Gubernamentales y Sociales de la Población de la Tercera Edad en Colombia. Tesis de Grado. Cali: Universidad Autónoma de Occidente. Cali, 92 pp. 